\title{
Commentary \\ Extracorporeal life support for status asthmaticus: the breath of life that's often forgotten
}

\author{
Nana Ekua Coleman and Heidi J Dalton
}

Department of Critical Care Medicine, Children's National Medical Center, The George Washington University School of Medicine, Washington, DC 20010, USA

Corresponding author: Heidi J Dalton, hdalton@cnmc.org

Published: 28 April 2009

Critical Care 2009, 13:136 (doi:10.1186/cc7757)

This article is online at http://ccforum.com/content/13/2/136

(c) 2009 BioMed Central Ltd

See related research by Hebbar et al., http://ccforum.com/content/13/2/R29

\begin{abstract}
Status asthmaticus continues to be significant cause of intensive care admission, morbidity, and mortality in pediatric populations. Furthermore, despite improved outpatient management and broader use of controller medications, patients with severe status asthmaticus account for a notable proportion of these admissions. There is variability in management and outcomes between institutions; however, early and aggressive management to avoid respiratory failure is paramount. In those patients who progress to develop severe respiratory failure, extracorporeal life support (ECLS) can be a life-saving therapy. Here, we briefly overview the use of ECLS for status asthmaticus, as reported through the Extracorporeal Life Support Organization, including the specific institutional experience at Children's Healthcare of Atlanta at Egleston, and consider how earlier initiation of ECLS may benefit patients with severe status asthmaticus refractory to conventional medical therapy.
\end{abstract}

The Extracorporeal Life Support Organization (ELSO) registry reports 64 uses of ECLS during the period from 1986 to 2007, including 13 patients from Children's Healthcare of Atlanta at Egleston, as presented by Hebbar and coworkers [1] in their discussion of extracorporeal life support (ECLS) for refractory severe status asthmaticus (SSA). Overall survival was $100 \%$ in the Egleston cohort and $92 \%$ in the remaining 51 patients reported in the ELSO registry. It is of interest to note that all of the 13 survivors from the Egleston series had no reported neurological sequelae. This outcome is comparable to the $6 \%$ incidence of neurological complications (seizure and intracranial hemorrhage) observed in the larger group; however, in neither group were neurological sequelae correlated with overall outcome.

The observed characteristics of patients receiving ECLS for SSA was similar between the two groups. In general, patients who received ECLS had a median age of 10 years; before
ECLS they had a serum $\mathrm{pH}$ of less than 7.0 , an arterial carbon dioxide tension $\left(\mathrm{PaCO}_{2}\right)$ above 120 torr, and an arterial oxygen tension above 50 torr. The percentage of patients in whom venovenous (VV) cannulation was used was higher in the Egleston group than in the ELSO group (92\% versus $82 \%$ ), but over time more patients underwent VV cannulation. Despite these demographic characteristics, there were no statistically significant differences in survival and outcome between the two groups.

Although previous studies [2,3] have considered outcomes for ECLS for status asthmaticus in adults, the work of Hebbar and coworkers [1] is the first to discuss pediatric outcomes comprehensively. In our opinion, the clinical outcomes observed in both the Egleston and the larger ELSO series support the early use of ECLS in status asthmaticus, and moreover they highlight the need to define clinical parameters that should prompt strong consideration of ECLS. We advocate a system that first identifies patients at high risk for developing refractory status asthmaticus: those with history of multiple intubations and/or respiratory failure requiring intubation within 6 hours of admission; those with hemodynamic instability and/or neurological impairment at time of admission; and those with a duration of respiratory failure greater than 12 hours despite maximal medical therapy, as defined by institutional availability. We are reluctant to propose distinct serum $\mathrm{pH}$ and $\mathrm{PaCO}_{2}$ values as pre-ECLS criteria, given there is no correlation of these factors with survival. However, in general, sustained $\mathrm{PaCO}_{2}$ retention above $100 \mathrm{mmHg}$ and persistent serum $\mathrm{pH}$ below 7.0 should warrant discussion of ECLS.

Where available, ECLS referral should be made early and decisively. There are clear risks associated with ECLS, but

$\mathrm{ECLS}=$ extracorporeal life support; ELSO = Extracorporeal Life Support Organization; $\mathrm{PaCO}_{2}=$ arterial carbon dioxide tension; SSA $=$ severe status asthmaticus; $\mathrm{VV}=$ venovenous. 
avoidance of cardiopulmonary arrest, attenuation of lung injury associated with prolonged mechanical ventilation at high pressures, and reduction in the systemic toxicities associated with medical therapies for SSA should be among the goals of therapy with ECLS for refractory status asthmaticus. Cannulation strategies should be patient specific. However, the recent trend toward VV cannulation for SSA reported in the ELSO registry is noted and reasonable, because this mode allows for preservation of arterial vascular integrity and provides sufficient pulmonary support during the acute period of illness in those patients without cardiovascular compromise. The recent availability of percutaneous insertion kits and new, double-lumen single cannulas that range in size from 13 to $32 \mathrm{Fr}$ make implementation of $\mathrm{VV}$ support in children and adults potentially even easier and safer. If ECLS were considered at the time of presentation based on the aforementioned criteria, it is possible that patients could require shorter ECLS courses, because they would not yet have developed the severe respiratory, hemodynamic, and metabolic derangements that may prolong the duration of ECLS. The experience from Children's Healthcare of Atlanta at Egleston successfully demonstrates the use of ECLS as an adjunctive strategy for managing SSA. The work there is both important and relevant, because it illustrates consistent, positive outcomes over time with use of this therapy.

SSA is a self-limited, reversible disease process, which - if treated aggressively at the onset - does not have to be fatal. It is of interest that although use of ECMO in adults is still a rarity, a recent review of adult ECMO also focused on use in status asthmaticus [4]. Both the adult and pediatric studies have found small numbers of patients, but it is unknown whether this reflects the small numbers of patients who develop refractory status asthmaticus or just the fact that ECMO is rarely considered, no matter how bad the respiratory failure associated with asthma becomes. The authors might suggest that the latter is likely. With current technology, ECMO is safer and easier to perform than it has ever been. Perhaps raising the visibility of this technique as a support tool in severe asthma would decrease the mortality rate and improve patient care in children and adults alike.

\section{Competing interests}

The authors declare that they have no competing interests.

\section{References}

1. Hebbar KB, Petrillo-Albarano T, Coto-Puckett W, Heard M, Rycus PT, Fortenberry JD: Experience with use of extracorporeal life support for severe refractory status asthmaticus in children. Crit Care 2009, 13:R29.

2. Shapiro MB, Kleaveland AC, Bartlett RH: Extracorporeal life support for status asthmaticus. Chest 1993, 103:1651-1654.

3. Kukita I, Okamoto K, Sato T, Shibata Y, Taki K, Kurose M, Terasaki $\mathrm{H}$, Kohrogi $\mathrm{H}$, Ando M: Emergency extracorporeal life support for patients with near-fatal status asthmaticus. Am J Emerg Med 1997, 15:566-569.

4. Mikkelsen ME, Woo YJ, Sager JS, Fuchs BD, Christie JD: Outcomes using extracorporeal life support for adult respiratory failure due to status asthmaticus. ASAIO J 2009, 55:47-52. 